\title{
THE UNMANNED MISSION AVIONICS TEST HELICIOPTER - A FLEXIBLE AND VERSATILE VTOL-UAS EXPERIMENTAL SYSTEM
}

\author{
Dr. H.-W. Schulz \\ ESG Elektroniksystem- und Logistik-GmbH, D-82256 Fürstenfeldbruck, Germany - hws@esg.de
}

KEY WORDS: UMAT, Unmanned Mission Avionics Test Helicopter, Unbemannter Missionsausrüstungsträger, VTOL, UAS, UAV, Experimental, Safety Concept, Cassette Solution

\begin{abstract}
In 2009 ESG procured a VTOL-UAS to offer an unmanned test helicopter for special applications of military and civil customers. These applications cover a wide spectrum from R\&D programs for the military customer to special services for the civil customer. This paper focuses on the technical conversion of a commercially available VTOL-UAS to ESG's Unmanned Mission Avionics Test Helicopter (UMAT), its concept and operational capabilities. At the end of the paper, the current integration of a radar sensor is described as an example of the UMATs flexibility. The radar sensor is developed by the Fraunhofer Institute for High Frequency Physics and Radar Techniques (FHR). It is integrated by ESG together with the industrial partner SWISS UAV.
\end{abstract}

\section{THE UNMANNED MISSION AVIONICS TEST HELICOPTER}

\subsection{Introduction}

In 2009 the Elektroniksystem- und Logistik-GmbH (ESG) invested in a vertical takeoff and landing (VTOL) unmanned aircraft system (UAS). This started the development of the Unmanned Mission Avionics Test Helicopter - the UMAT. Based on the Swiss UAV "NEO S-350 Twin" the UMAT shall become a flexible, versatile experimental system for use by ESG and its customers. The UMAT's capabilities are intended to develop in several phases, named Operational Capability Levels (OCL). This approach, documented later in this paper, has already been approved by the military certification authorities. Enormous value is added to the UMAT by integrating variable experimental payload in Operational Capability Level 1 (OCL1) configuration and mission and flight guidance avionics in an OCL2 configuration respectively. These Operational Capability Levels allow independent analysis that goes along with the development of current and future research and technology programs.

\subsection{UMAT Objectives}

The UMAT has special importance for the continuation of the current research and technology project Manned-Unmanned Teaming (MUM-T, now called MiDEA), where an unmanned platform is needed to emulate the future unmanned target system of the Manned-Unmanned Army Helicopter Cooperation Concept. The UMAT is supposed to be this target system emulating platform. The continued use of the UMAT for experimental analysis in the field of research and technology is already planned. These include:

- analysis of certification requirements and processes for unmanned aircraft systems, of essential system functions and operating procedures as well as the process of showing compliance

- $\quad$ independent verification of VTOL-UAS and components performance

$\circ$ performance (payload/endurance) automatic takeoff and landing (ATOL), sense and avoid (S\&A), sensor- / data link occlusion

o operational suitability of alternative commercial of the shelf mission avionics and system components (sensors, data link, Human Machine Interface) $\circ$ environmental tests

- concepts of operation SAATEG VTOL Marine / Army and MUM-T

- independent development accompanying experiments within current and future research and technology programs, such as

- MUM-T (Manned-Unmanned Teaming)

- MiDEA (mission escort by drones for exploration and reconnaissance)

- MiRA-T (crew assistance function for optimized crew resource management in the cockpit, integration of UASs)

- SAAFu (Sense \& Avoid Assistance Function for the UAS-Operator)

- Human Machine Interface for ground control station with assistance functions for mission planning and UAV guidance and control

- development and analysis of autonomous mission management system functions

To support those research and technology programs, which often use noncertified prototypes, the UMAT itself must be a certified, flexible, unmanned experimental system capable of carrying noncertified experimental equipment. Therefore ESG and its industrial partner Swiss UAV together with civil and military certification authorities started the definition and setup of a certification process for its VTOL-UAS test helicopter. But this paper focuses on the technical concept of the UMAT.

\subsection{UMAT Concept}

The UMAT (shown in Fig. 1) is based on the VTOL UAS "NEO S-350 Twin", developed by Swiss UAV.

The basic performance data of the Swiss UAV NEO S-350 Twin are:

- rotor diameter: $350 \mathrm{~cm}$

- dimensions: 325 x 105 x $94 \mathrm{~cm}$

- maximum takeoff weight / payload: $125 \mathrm{~kg} / 30 \mathrm{~kg}$ (depending on fuel)

- power plant: 2 x $30 \mathrm{~kW}$ turbine

- max speed: up to $120 \mathrm{~km} / \mathrm{h}$

- max endurance: up to 3.5 hours

- STANAG 4586 compatible ground control station 


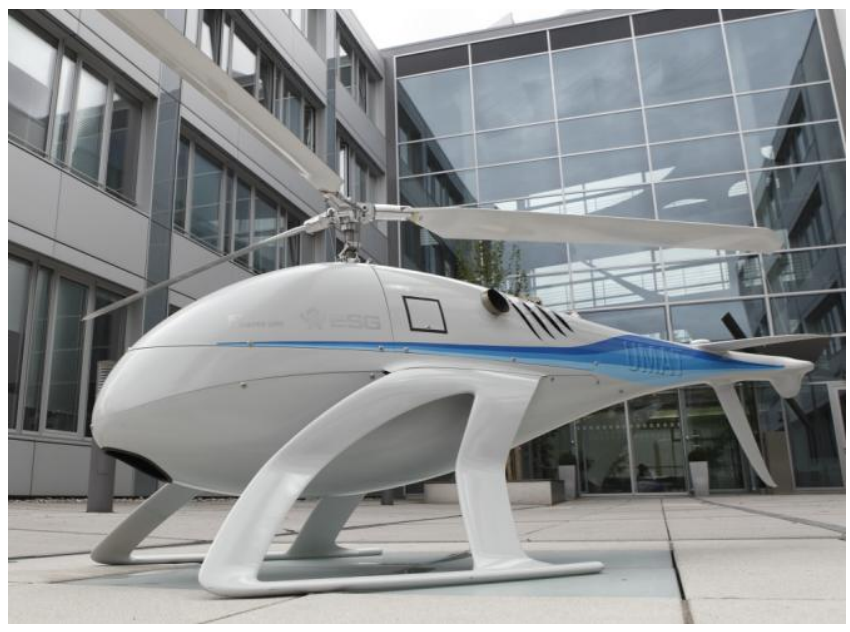

Fig. 1 Unmanned Mission Avionics Test Helicopter based on Swiss UAV NEO S-350 Twin

This modification allows transporting experimental equipment, which does not have to be certified, if considered as a nonessential payload system, not interfering with the UMAT platform. Instead, a simplified qualification is considered sufficient. The experimental equipment can be classified into a permanent part which is used for all experiments and a variable part. The latter is the operationally relevant part of the UMAT payload.

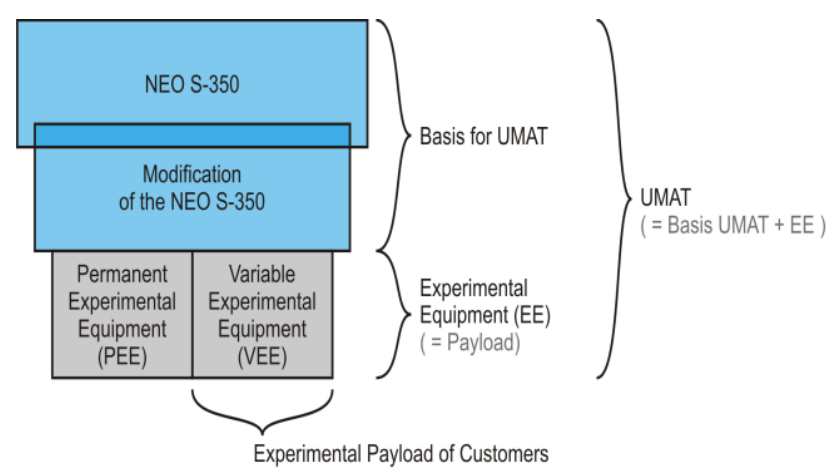

Fig. 3 Unmanned Mission Avionics Test Helicopter based on Swiss UAV NEO S-350 Twin

The following notional example should clarify this: The prototype of a magnetic field sensor - to be tested with the UMAT - is the variable experimental equipment (VEE). This sensor needs navigation data from the UMAT to function properly. This data can be provided by the permanent experimental equipment (PEE): The mission avionics core (MAC) of the permanent experimental equipment, which can be customized for every experiment, receives the navigation data from the basic UMAT and prepares it for the magnetic field sensor in an adequate way.

The UMAT development concept includes the type certification of the UMAT and any of its later capability levels. The implementation of permanent experimental equipment and any variable experimental equipment shall not affect the validity of aforementioned type certification of the UMAT basis and therefore shall require equipment qualification and compatibility checks only to proof non-interference of the equipment with safety objectives. The concept of integration of any experimental equipment (EE) shall be such that clear and

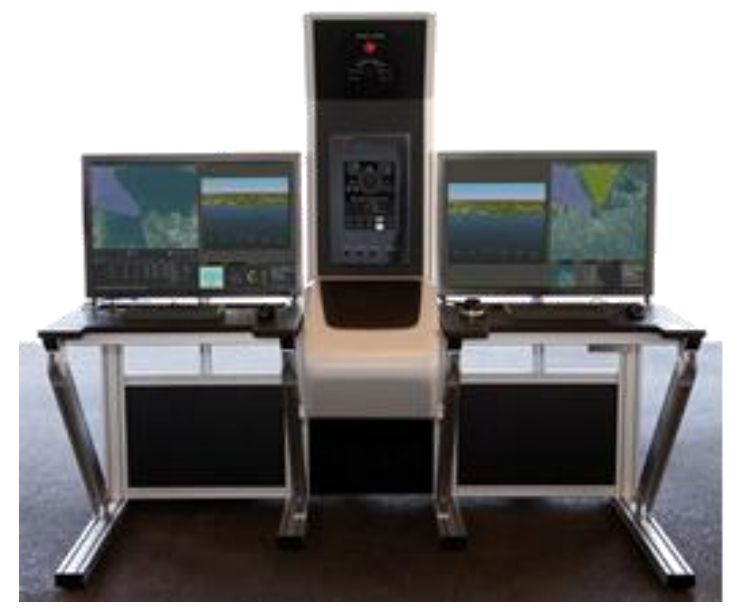

Fig. 2 Current Ground Control Station of the Swiss UAV NEO S-350 Twin

unambiguous UMAT hardware interfaces and performance requirements will be defined. These manifest as an encapsulated payload cassette (see Fig. 2 and Fig. 3) carrying the permanent and any suitable variable experimental equipment. This will ensure that any payload - when specified, designed and qualified within the defined interface and performance boundaries of the UMAT will not impair the certified safety and performance objectives of the certified UMAT, hence making a supplemental type inspection unnecessary.

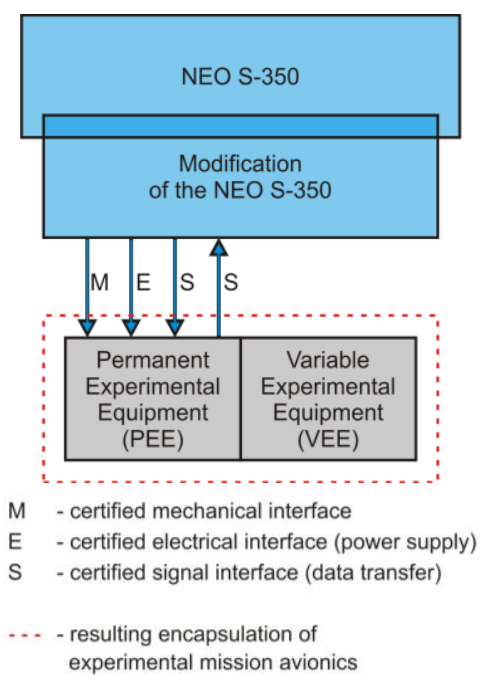

Fig. 4 Payload Cassette Safety Concept to encapsulate Experimental Equipment

\subsection{UMAT Operational Capabilities Level}

The planned use of the UMAT calls for different capabilities. The realization of those capabilities can be structured in phases. The UMAT operational capabilities and utilization concept foresees a 4 level concept as outlined in Fig. 6.

\section{Green Aircraft - UMAT (GA)}

The UMAT (GA) shall allow the demonstration of the technical capabilities (function, safety and performance) to operate the UAV with the main objectives to:

- commence initial flight test controlled via the UAS ground control station with the purpose of familiarization and evaluation of the UAS basic function, safety and performance

- conduct any flight test program to support the compliance demonstration required for the certification process 


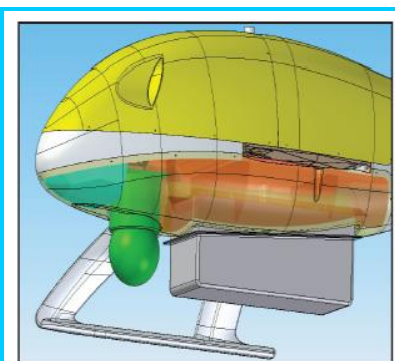

top cover and cassette bottom encapsulate the experimental equipment, and provide safety NEO's payload rail against mechanical failure and sys-tem electromagnetic interference top cover using the

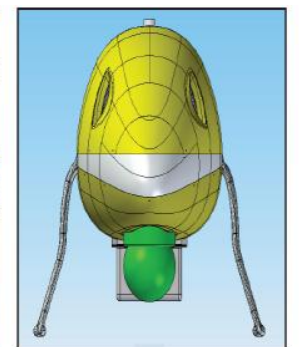

mounting of cassette

Fig. 5 Payload Cassette Safety Concept to encapsulate Experimental Equipment

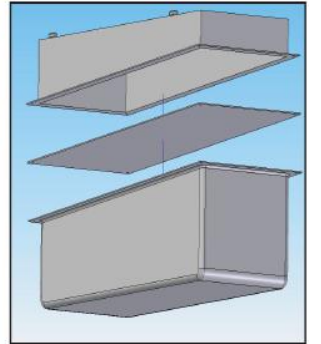

lateral positioning of experimental equipment pay- remaining ground load cassette enables adjustment of air-craft centre clearance of $90 \mathrm{~mm}$ of gravity $( \pm 10 \mathrm{~mm})$

\begin{tabular}{|c|c|c|c|}
\hline Gre & en Aircraft & - & onboard recording of system and flight data \\
\hline 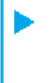 & $\begin{array}{l}\text { Assessment of UMAT performance and capabilities } \\
\text { without interfering with the base system }\end{array}$ & & \\
\hline Op & erational Capability Level 1 & 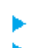 & integration of varying experimental equipment \\
\hline$>$ & Unmanned Mission Avionics Test helicopter & 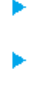 & $\begin{array}{l}\text { lead access to UMAT (GA)-onboard-sensors for } \\
\text { UMAT experimental equipment } \\
\text { ESG avionics (Permanent Exp. Equ. / PEE) } \\
\text { drive Variable Experimental Equipment (VEE) }\end{array}$ \\
\hline Op & erational Capability Level 2 & - & replacement of human operator with ESG \\
\hline 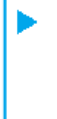 & $\begin{array}{l}\text { MMC-access to flight guidance and flight } \\
\text { management systems of the base system using the } \\
\text { existing UCS }\end{array}$ & - & $\begin{array}{l}\text { Mission Mgmt. Computer (MMC, on ground) } \\
\text { e.g. MUM-T II / MiDEA Flight Test I: } \\
\text { sound safety concept }\end{array}$ \\
\hline Op & erational Capability Level 3 & - & replacement of UCS with ESG Mission \\
\hline & $\begin{array}{l}\text { MMC direct onboard access to flight guidance and } \\
\text { flight management systems of the base system }\end{array}$ & - & $\begin{array}{l}\text { Management Computer (MMC, on board) } \\
\text { e.g. MUM-T II /MiDEA Flight Test II }\end{array}$ \\
\hline
\end{tabular}

Fig. 6 UMAT levels of operational capabilities
These objectives are supported by the NEO onboard recording capabilities, which $\log$ flight and system states. Worth mentioning is the integrated simulation mode of the NEO, which allows to simulate the complete flight envelope of the UMAT inside the laboratory.

UMAT Operational Capability Level 1 (OCL1)

The most important of the UMAT Operational Capability Level is UMAT (OCL1). Modifying the UMAT Green Aircraft using the payload cassette concept shall facilitate an accelerated process to rapidly introduce any variable experimental equipment into the UMAT (OCL1) without making a supplementary type investigation necessary. Only equipmentqualification and vehicle compatibility shall be applied for a change of experimental equipment. The intention is to reduce the administrative effort to an acceptable minimum without jeopardizing overall airworthiness and safety. The result will be the certified, versatile, flexible, unmanned experimental system capable of carrying noncertified experimental equipment.

The UMAT Operational Capability Level 1 shall include the integration of the payload cassette with the main objectives to:
- $\quad$ conduct a flight test program to demonstrate compatibility and non-interference of the payload cassette including the permanent experimental equipment (as part of the recertification process after modification of the UMAT)

- $\quad$ operate the UMAT with variable experimental equipment

- $\quad$ operate variable qualified experimental equipment installed in the UMAT(OCL1) payload cassette with a UMAT(OCL1) Certificate of Conformity (CoC)

- $\quad$ use the read-only access of the permanent experimental equipment to vehicle avionic / system data to provide services to variable experimental equipment

- control the variable experimental equipment using the permanent experimental equipment avionics

For the qualification of the experimental equipment inside the payload cassette a qualification process must be defined. This process has to be approved by the certification authority. Successful completion of this process allows to quickly return to flight operations of the UMAT after each single modification of the experimental equipment. 
One example of using the UMAT (OCL1) is shown in Fig. 7. To test meteorological payload no connection between experimental equipment and safety critically UMAT avionics is necessary. Mechanical modifications are limited to the payload bay and are not compromising the certification of the UMAT basis. Should this not be sufficient - for example because a nose boom is needed for wind measurement - a supplemental type certificate becomes necessary.

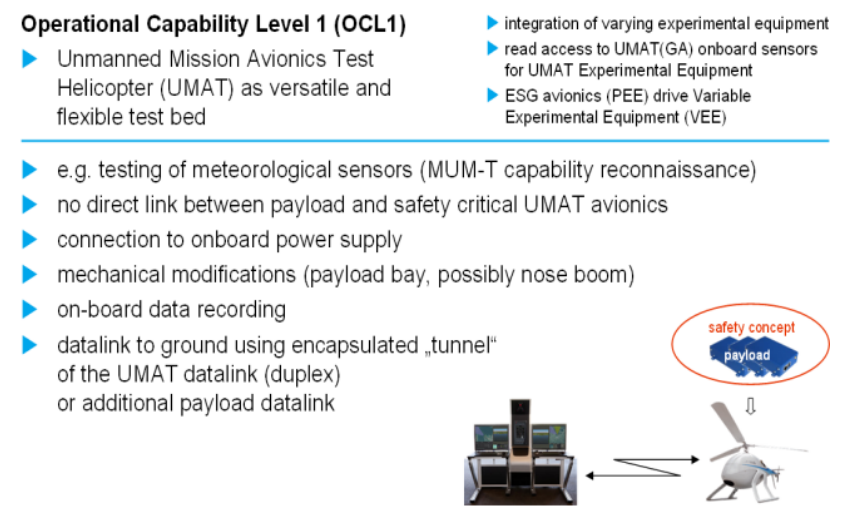

Fig. 7 Depiction of UMAT Operational Capability Level 1

\section{UMAT Operational Capability Level 2 (OCL2)}

UMAT (OCL2) shall include the implementation of an experimental ground based Mission Management Computer (MMC) in addition to the certified ground station operator. This would be used to test autonomy increasing functions using UMAT flight experiments. It is expected that the safety concept which - for this Operational Capability Level - has still to be detailed, will encapsulate the certified ground control station in such a way, that the signals of the experimental mission management computer can be monitored and corrected at any time. This capability level allows to:

- continue the agreed flight test program to obtain a supplemental type certificate for this UMAT OCL

- demonstrate the suitability of the Mission Management Computer (MMC) for "autonomous operation" as a prerequisite for an UMAT integrated MMC as intended by UMAT Operational Capability Level 3 (OCL3)

- operate the MMC equipment installed in the UMAT ground control station with a Certificate of Conformity

Using these operational capability first flight tests of the Manned-Unmanned Teaming program can be conducted by deploying the unmanned sensor platform (UMAT) from the manned command helicopter (MAT/ manned Mission Avionics Test helicopter). At this stage no direct link between UMAT and MAT exists. All high-level commands of the UAV operator on board the MAT will be linked to the experimental mission management computer on the ground. The signals of that experimental avionics system have to pass through the certified ground control station, manned by qualified personnel.

\section{UMAT Operational Capability Level 3 (OCL3)}

A consequent continuation of the aforementioned UMAT Operational Capability Level 2 is the integration of the tested mission management computer into the onboard experimental equipment of the UMAT, representing the last of the three modification steps. A direct writing link between the experimental equipment and the certified UMAT Green Aircraft avionics allows the functions to be tested to directly influence the UMAT. Clearly, the safety concept for this case has to be specially designed. One possible solution is to use the certified ground control station to monitor the onboard mission management computer at all times, to correct any errors or to deactivate the test equipment.

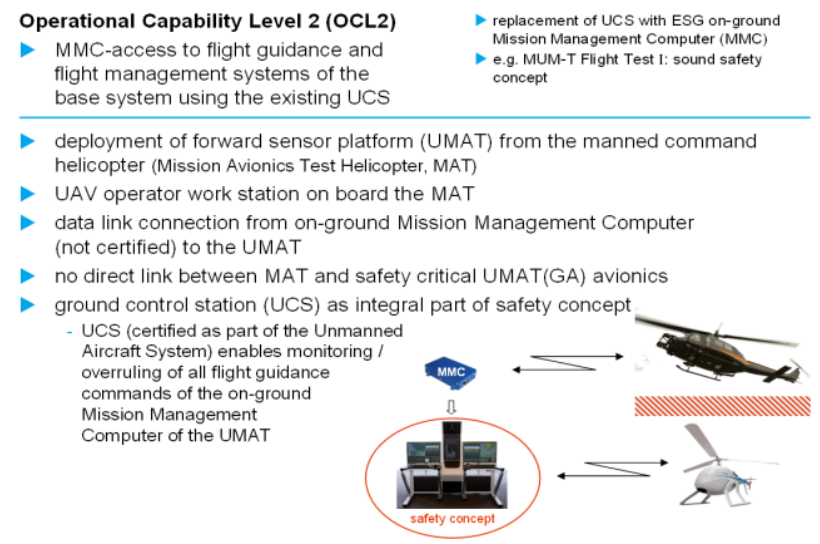

Fig. 8 Depiction of UMAT Operational Capability Level 2

The UMAT Operational Capability Level 3 makes extensive Manned-Unmanned Teaming experiments possible, creating a direct link between the experimental mission management computer onboard the UMAT and the manned command helicopter (MAT / manned Mission Avionics Test helicopter).

UMAT (OCL3) shall include the implementation of a Mission Management Computer into the variable experimental equipment of the UMAT UAV complementing the ground control station operator and includes operation to

- continue the agreed flight test program to obtain a supplemental type certificate for this UMAT Operational Capability Level

- demonstrate the capability for Mission Management Computer controlled autonomous operation

- test various autonomy improving functions on board the mission management computer

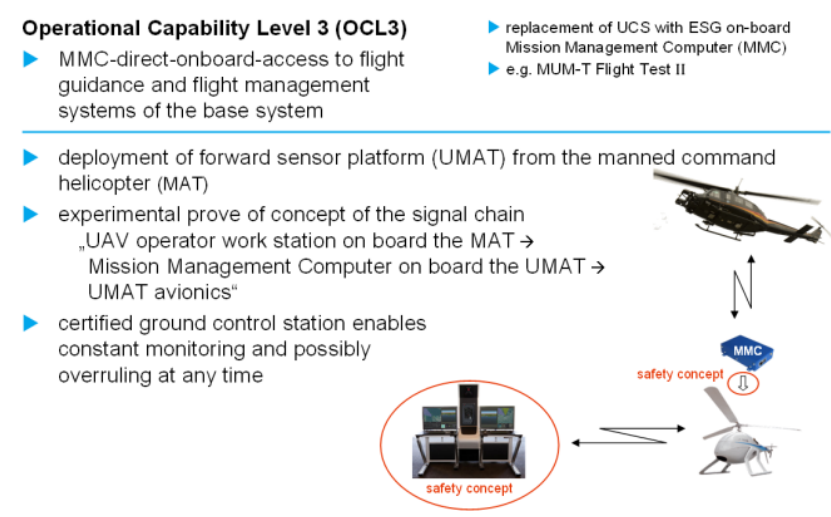

Fig. 9 Depiction of UMAT Operational Capability Level 3 


\section{INTEGRATION OF A SYNTHETIC APERATURE RADAR (SAR) SENSOR}

\subsection{Integration of the Sensor}

The Fraunhofer Institute for High Frequency Physics and Radar Techniques (FHR) develops a Synthetic Aperture Millimetre Wave Radar, operating at $94 \mathrm{GHz}$ which is called SUMATRA. ESG and FHR combined forces to analyse the suitability of FHRs high performance radar within the special environment of small VTOL-UAS.

The integration includes the SAR-front end with its sending and receiving antennas and a high precision navigation system of low weight and volume (see Fig. 10).

The SUMATRA Radar is the first Experimental Equipment using the UMAT. Due to the high flexibility of the UMAT integration was successfully completed within a few days at the end of July 2011.

\subsection{Test Flights}

After thorough ground based interaction and interference tests the first flight on July $7^{\text {th }}$ focused on demonstrating safe flight of the UMAT and basic operation of the Radar.

The next flight will use a different operating range providing more flexibility in flight path planning: Long straight legs are needed to evaluate the SARs performance. Several test flights in different weather conditions are planned.

These flights will demonstrate a first part of the true potential of the UMAT as a flexible, unmanned experimental system capable of carrying miscellaneous experimental equipment.

\subsection{SUMATRA Campaign Storkow}

In mid August ESG and Fraunhofer FHR started a test campaign with the SUMATRA sensor. The test area - a military restricted training site in Storkow is located south of Berlin. The site offered tests above rural and urban environment.

These recently made flights could prove the overall functionality of the radar. The picture below (Fig. 13) is one of the early results. This Fraunhofer Institute SAR Sensor generated image is not yet a „Radar Picture“. Each vertical line of pixels of this real time data projection represents the amount of reflected energy. Each new line of pixels represents another data acquisition cycle and hence a new instance of time. No postprocessing has been applied yet. The postprocessing of this raw data will account for many effects, but mainly it will take into account the six-degree-of-freedom movement of the helicopter as measured by the high accuracy inertial navigation system onboard the UMAT. In a further step a digital terrain model will be included in the processing as well. Using this information the Fraunhofer FHR will create a georeferenced image in postprocessing.

Since the effects of both these motions and the changing terrain are still included in the raw data, the image shown is not sharp, rather blurred and contains several artefacts.

But the first impression of the picture shows several objects of the captured scenario already, like 3 Patriot Launcher, the UMAT control station ('above' the launchers) and the radar receiving terminal ('right' of the launchers).

After the successful integration of the radar sensor SUMATRA into the UMAT and the early tests, future test flights and evaluation will investigate the operational applicability of the sensor onboard tactical VTOL-UAVs.

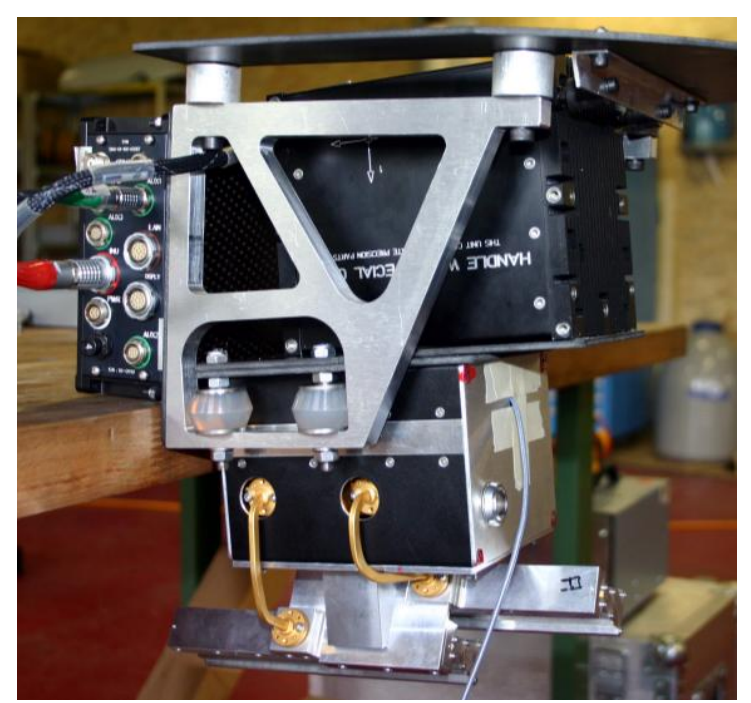

Fig. 10 Compact sensor package consisting of SAR-frontend with antennas, IMU and navigation computer

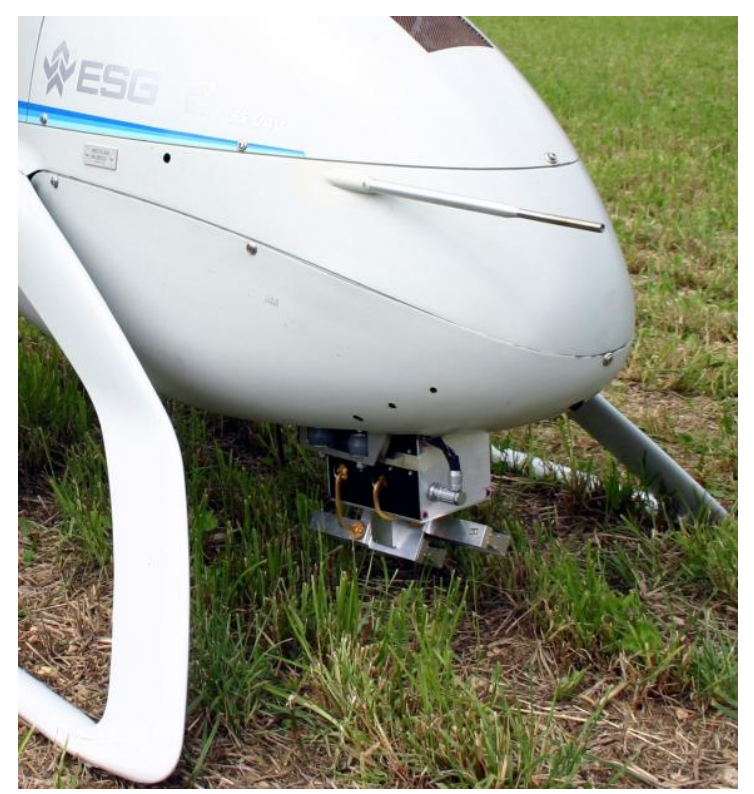

Fig. 11 SAR sensor package mounted on UMAT

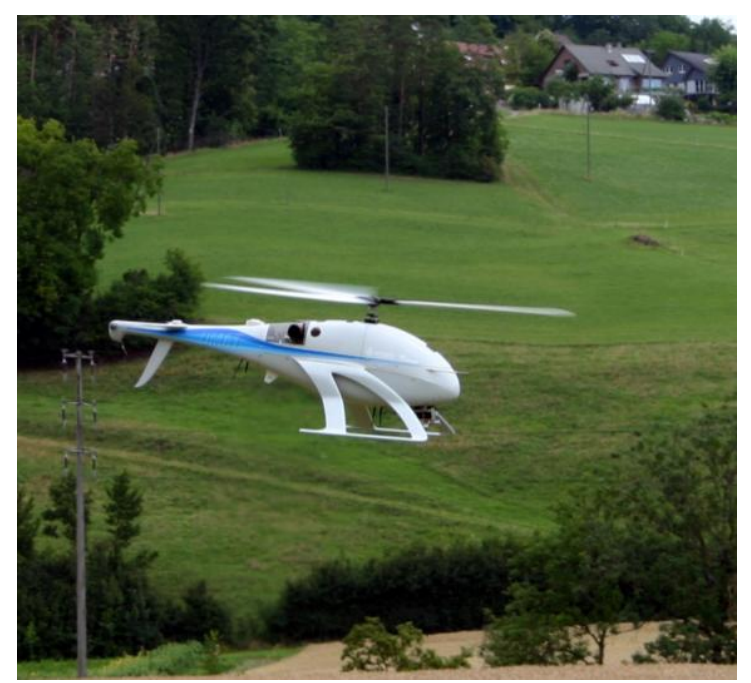

Fig. 12 First flight of SAR sensor package using the UMAT 


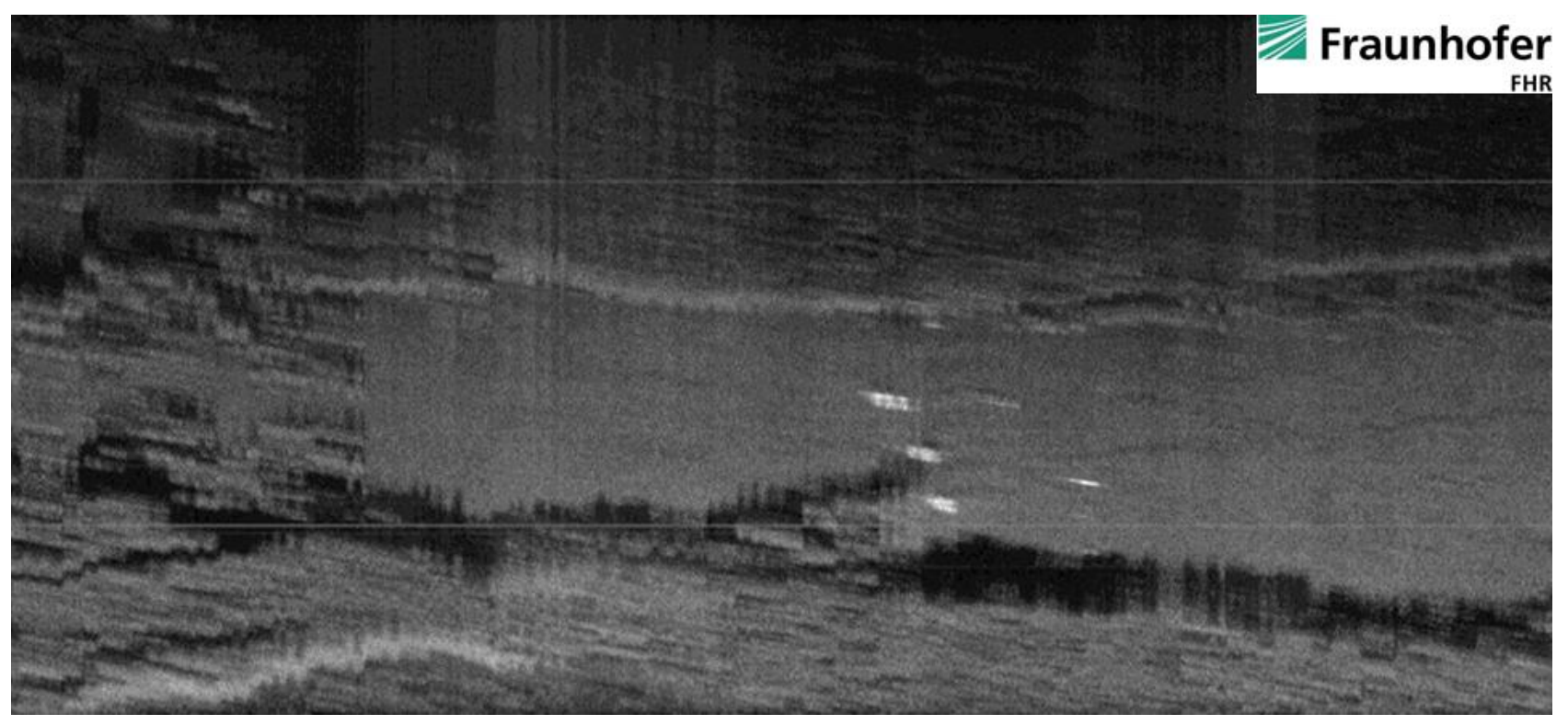

Fig. 13 Screenshot of the real time projection of the received reflected energy over time

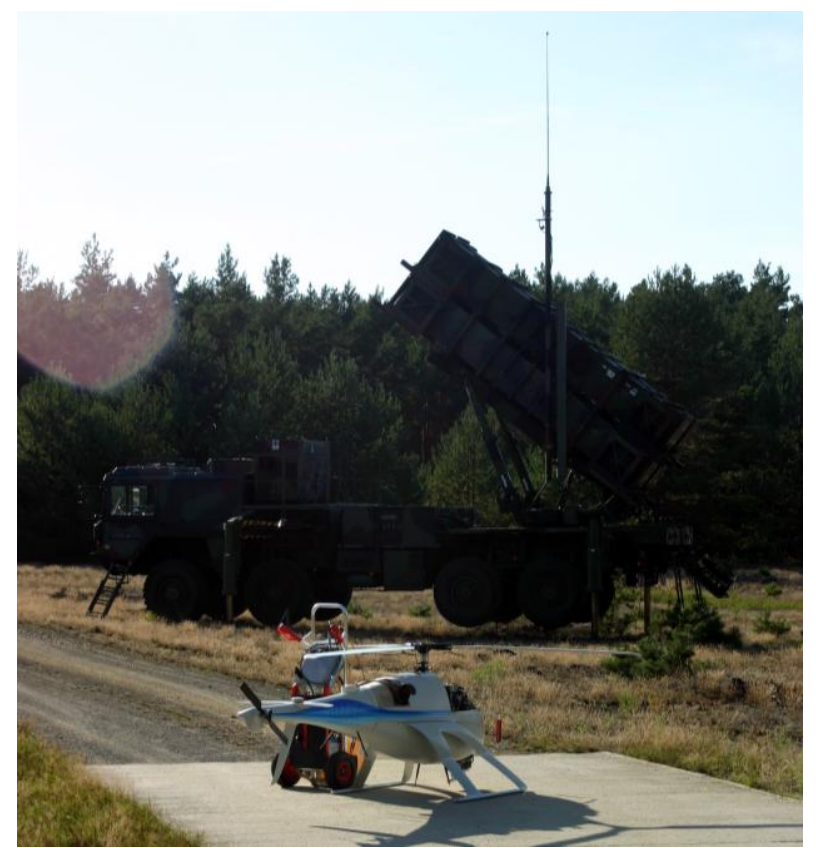

Fig. 14 UMAT with Patriot Launcher in the back

\subsection{Conclusion}

Based on a commercially available VTOL UAS the concept of a versatile flying experimental testbed for special applications of military and civil customers was conceived. The UMAT offers / will offer the following service capabilities:

- $\quad$ highly versatile, experimental platform for easy integration of varying payloads

- $\quad$ strict separation of experimental avionics and certified UMAT avionic system represents a unique safety concept

- $\quad$ safety concept enables rapid integration and simplified qualification of experimental Hard- \& Software

- extensive, simultaneous onboard / onground data acquisition and recording of system and state data for post processing

- experimental ESG onboard Mission Management Computer (MMC) provides capacity for additional autonomous system functions (e.g. onboard mission replanning, sense $\&$ avoid, ...)

- UMAT UAV Control Station (UCS) with integrated MMC-clone enables reliable monitoring and overruling of all UMAT flight guidance modes

- UMAT can be integrated into the unique ESG MannedUnmanned Test Environment (Manned Mission Avionics Test Helicopter / MAT and UAS Mission Training Simulation / UMiTS)

- combined test flights of the UMAT and the proven MAT enable special experimental analysis in realistic, operational scenarios, e.g. Manned-Unmanned Teaming

Promising results could be obtained with an imaging radar sensor for VTOL UAS applications. The success of this first experimental equipment integration confirms the UMAT concept. In the future ESG will test various payloads intended for VTOL UAS. 